\title{
ANALYSIS OF MULTI-HOP RELAY ALGORITHM FOR EFFICIENT BROADCASTING IN MANETS
}

\author{
Dipali K. Dakhole ${ }^{1}$, Archana R. Raut ${ }^{2}$ \\ ${ }^{1}$ Computer Science and Engineering Department, G.H. Raisoni College of Engineering, Nagpur, India \\ ${ }^{2}$ Computer Science and Engineering Department, G.H. Raisoni College of Engineering, Nagpur, India
}

\begin{abstract}
Broadcasting means communicating information from one to all or many to all nodes in a network. It is widely used in many MANET routing protocols. Flexible and distributed MANETs are robust and rapidly deployable/reconfigurable, so they are highly appealing for a lot critical applications, like deep space communication, disaster relief, battlefield communication, outdoor mining, etc. To improve such limitation for a more efficient utilization of limited wireless bandwidth, this paper proposes a more general probing based multi-hop relay algorithm with limited energy consumption. A general theoretical framework is further developed to help us to understand that under different network size, how we can benefit from multiple observations in terms of per node PDR, the expected end to end delay and limited energy consumption.
\end{abstract}

Keywords: MANETs, Broadcasting, Multi-hop relay, end-to-end delay, PDR, etc. $* * *$

\section{INTRODUCTION}

In mobile ad hoc networks (MANETs), as nodes move around randomly, network topology varies dramatically and there may not exists an end to end path at any given instant. If point to point communication is there between the nodes, then surely MANETs gives better throughput. The traditional routing based protocols such AODV, DSR, etc. fail to function properly as they require simultaneous availability of number of links [1] [2]. Two-hop relay algorithm is able to provide a flexible control of both throughout and packet delay for the challenging MANETs. But under such routing scheme, a packet has been transmitted either through direct transmission from source to destination or by two-hop via an intermediate relay node, which first receives packet from source and then forward it to the destination [3],[4]. But here source and destination must be one relay node apart from each other.

Every node present in MANET can be considered a router. The source node utilizes the intermediate nodes to transmit the message towards the destination node if a source node fails to transmit a message reliably to its destination node. MANET networks propose reliability, bandwidth and battery power and have erratic traits like topology, signal strength and transmission routes. Transmission algorithms and procedures are supposed to be very light to save energy and bandwidth in computation and storage necessities. A lot of primary work has been done to analyze the packet end-to-end delay and throughput. Regardless of much research activity on the delay performance study of multi -hop relay MANETs in last several decades, the important issue of end-to-end delay modeling in multi-hop relay MANETs remains a technical challenge. In multi-hop relay MANETs it is also mandatory to deliver the packet reliably without any loss of data. In multihop relay network system, it is very difficult to choose optimal path to deliver the data to destination node. Hence sending node will try for all possible paths, hence it will consume a lot of energy. To balance the energy constraint is one of the major challenge in multi-hop relay MANETs.

In this paper, research conducted to address issues in multihop relay selection, delay minimization, energy management and reliable packet delivery during broadcasting is described. The paper is organized as follows. Section 2 reviews related work. Section 3 describes briefly the proposed efficient technique for broadcasting. Section 4 analyzes the proposed technique. Section 5 summarizes the paper and point out the areas of future work.

\section{RELATED WORK}

A very simple approach for broadcasting is flooding. In this technique, each node upon receiving a packet for the first time rebroadcasts it. Such a protocol works well in parse network and in networks with high mobility, but it is inefficient as multiple copies will be received by nodes in the network. A lot of research is done to improve this broadcasting technique [6]. In paper [7], the broadcast based network coding technique has succeed in improving the packet delay and throughput, but the results has shown that the actual protocols are still far from achieving the possible lower bound. A cross session broadcast technique has been proposed in paper [5]. The results have shown that this protocol has improved the packet delay, PDR and throughput as it has divided the network into generations, however the management of these generations becomes more complex as their size increases. The last paper studied in 
literature has proposed an algorithm that is applicable for twohop relay algorithm [3]. It has improved packet delay and PDR drastically by considering static network scenario.

To the best of our knowledge, only few papers explicitly address multi-source wireless broadcast. Also a lot many work has been done by considering static network scenario only to get efficient results. Also many papers has focused on delay, PDR and throughput parameters only, the major constraint energy parameter is not considered along with these parameters. Hence more work need to be done to improve delay, PDR and energy parameters simultaneously.

\section{PROPOSED TECHNIQUE}

Broadcasting is the act of moving information from source to all other nodes or from more than one source node to all other nodes in the network. As we are considering multi-hop relay network, more than one intermediate node are considered during transmission of data in terms of packet. The main purpose of this algorithm is to optimize end-to-end delay, Packet delivery ratio (PDR) and data transmission energy of network.

\subsection{Definitions}

Throughput: As defined in usual way, the average number of bits that can be transmitted by each node to its destination per unit time is called as per node throughput. The sum of all per node throughput over all the nodes in a network is called as the throughput of the network.

Average Packet Delay: It is the time taken by packet to reach its destination after it leaves the source. The average packet delay of a network is obtained by averaging over all transmitted packets in the network.

Packet Delivery Ratio (PDR): It is the ratio of number of packets reached successfully at destination to the number of packets delivered by source node.

Data Transmission Energy: It is the energy of node required to carry data from source to its destination. The average data transmission energy of a network is obtained by averaging over all energy in the network.

\subsection{Network Model}

For implementation NS2 simulator is used. A network of 50 nodes is considered where all nodes are randomly moving. Each nodes location is identified by its $\mathrm{x}$ and $\mathrm{y}$ parameters. In this scenario every node is numbered from 0 to 49 and indicated by circles. Nodes $0,1,2,3,4,5$ which are in red color are considered to be broadcasting nodes. The remaining green colored nodes are data receiving nodes and will act as intermediate nodes too as shown in Fig.1. The simulation parameters of the network are given in below table.
Table 1 Simulation Parameters

\begin{tabular}{|l|l|}
\hline Queue Capacity & 50 packets \\
\hline Packet Size & $1000 \mathrm{kB}$ \\
\hline Packet Interval & $0.00001 \mathrm{Sec}$ \\
\hline Initial Energy of Node & $100 \mathrm{~J}$ \\
\hline Transmission Power & $0.2 \mathrm{~J}$ \\
\hline Sleep Power & $0.001 \mathrm{~J}$ \\
\hline Simulation Area & $300 \times 300$ \\
\hline Simulation Time & $10 \mathrm{Sec}$ \\
\hline
\end{tabular}

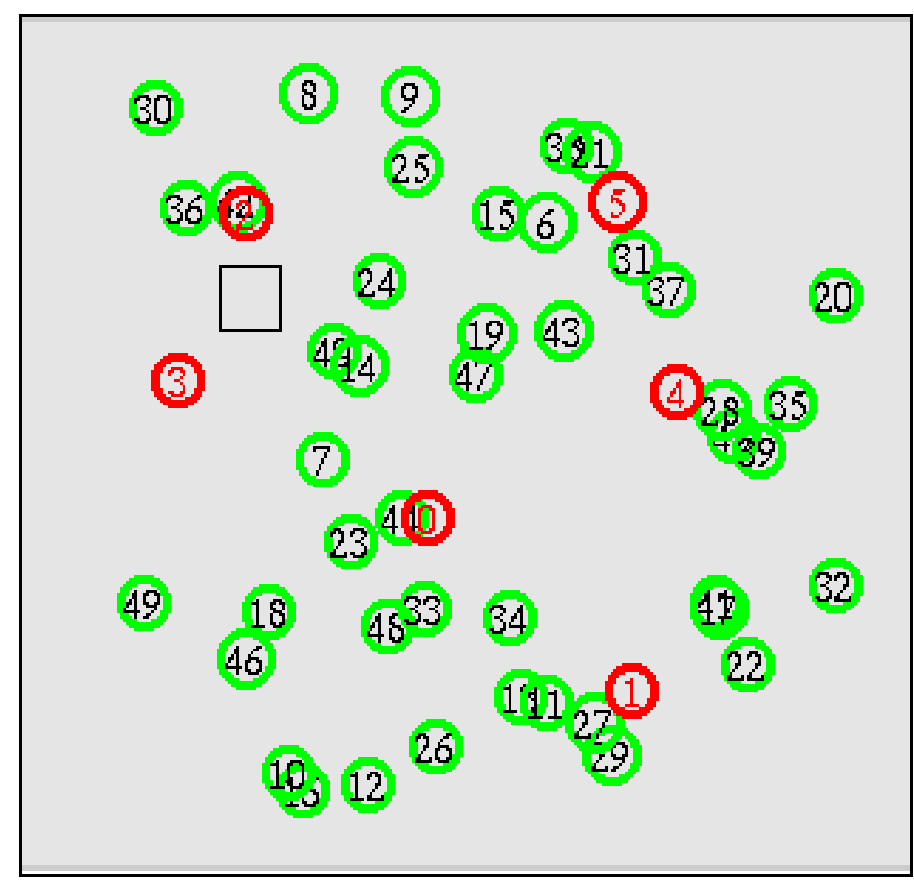

Fig 1 Network Formation in Multi-hop Relay MANETs

\subsection{Multi-hop Relay Algorithm}

As per considered in above network, there are total $\mathrm{n}$ (Number of nodes $\mathrm{n}=50$ ) distinct flows and each node can be a potential relay for other $\mathrm{n}-2$ flows (excluding the two flows originated from and destined for itself). To facilitate the operation of the Multi-hop relay routing protocol, each node is equipped with three types of First In First Served (FIFS) queues: one sourcequeue, one broadcast-queue and $n-2$ parallel relay-queues. These three types of queues are defined as:

Source Queue: It is a local queue storing the locally generated packets. It stores packets exogenously generated at $\mathrm{S}$ and destined for $\mathrm{D}$. These exogenous packets will be distributed out to relay nodes later in the FIFS way.

Broadcast Queue: Broadcast-queue stores packets from source-queue that have already been distributed out by $\mathrm{S}$ but have not been acknowledged yet by $\mathrm{D}$ the reception of them. 
Relay Queue: There are n-2 relay queues storing packets from other n-2 flows (one for each flow).

We denote source node as $\mathrm{S}$ and destination node as $\mathrm{D}$ as shown in Fig.2. We have considered a scenario where the source $\mathrm{S}$ and destination $\mathrm{D}$ use the push type of service for data transmission. S periodically sends locally generated packets to $\mathrm{D}$ via multiple intermediate nodes. While designing Multi-hop Relay algorithm, one problem is that $\mathrm{D}$ will receive multiple copies of packets. Hence to reduce this redundancy, every receiving intermediate relay node and $\mathrm{D}$ node is sending an acknowledgement ACK to the sending node immediately. Also to reduce data transmission energy, time allotted to send packet is $10 \%$ of slot and $90 \%$ of time is used to wait to receive ACK. Hence reliable packet delivery is also achieved.

Every time all queues, Source queue, Broadcast queue and Relay queues are updated. If packet is lost in between transmission process, then it is recovered from Relay queue of sender relay node so as to increase the throughput.

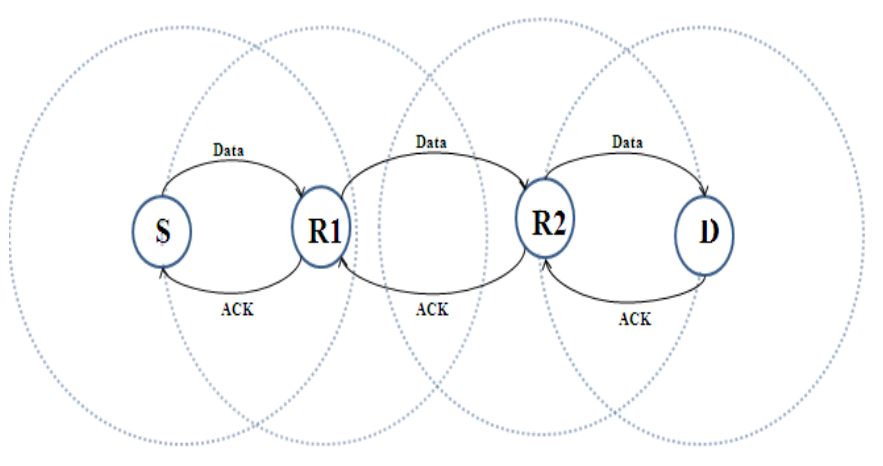

Fig 2 Multi-hop Relay Technique in MANETs

Every time $\mathrm{S}$ is selected as broadcasting node, it executes the following Algorithm 1.

Algorithm 1: Multi-hop Relay Algorithm

1. S checks whether its destination D is in the one-hop neighborhood;

2. if $\mathrm{D}$ is within the one-hop neighborhood of $\mathrm{S}$ then

3. $\quad$ S executes Procedure 1;

4. else

5. S randomly selects source-to-relay transmission or relay-to-destination transmission;

6. if $\mathrm{S}$ selects source-to-relay transmission then

7. $\quad$ S executes Procedure 2;

8. else

9. S executes Procedure 3;

10. end if

11. end if
Procedure 1: Source to Destination Transmission

1. S directly sends packet to $\mathrm{D}$ within $10 \%$ of time slot;

2. S waits for ACK within $90 \%$ of time slot;

3. if ACK not received then

4. $\mathrm{S}$ resends the packet to $\mathrm{D}$;

5. end if

6. S deletes packet from its source queue;

7. D updates the Broadcast queue;

Procedure 2: Source to Relay Transmission

1. S randomly search for nearby relay node $\mathrm{R}$ out of one hop neighbors;

2. S directly sends packet to $\mathrm{R}$ within $10 \%$ of time slot;

3. S waits for ACK within $90 \%$ of time slot;

4. if ACK not received then

5. $\mathrm{S}$ resends the packet to $\mathrm{R}$;

6. end if

7. S deletes packet from its source queue;

8. $\mathrm{R}$ updates the Relay queue;

Procedure 3: Relay to Destination Transmission

1. R search for destination node $\mathrm{D}$;

2. $\mathrm{R}$ directly sends packet to $\mathrm{D}$ within $10 \%$ of time slot;

3. R waits for ACK within $90 \%$ of time slot;

4. if ACK not received then

5. S resends the packet to $\mathrm{D}$;

6. end if

7. $\mathrm{R}$ deletes packet from its source queue;

This Multi-hop Relay algorithm has optimized delay performance, packet delivery ratio and data transmission energy of network in highly mobile multi-hop relay MANET. The further section will explain the results obtained in terms of delay, PDR and energy graph.

\section{SIMULATION RESULTS}

To validate the above proposed technique on expected end to end delay, PDR and energy remained after the execution of simulation, the following graphs are obtained.

\subsection{End-to-End Delay Validation}

The delay of network is calculated by following formula:

Delay of network $=\sum$ (Packet arrival time-sent time) $/$ number of connections.

A graph is plotted as delay of network against number of nodes. The nodes are varied from 10 to 100 and according to that the delay of network is plotted for existing $2 \mathrm{HR}$ routing technique and Multi-hop relay technique. The following graph shows that the proposed Multi-hop relay algorithm has optimized delay upto $72.79 \%$. 


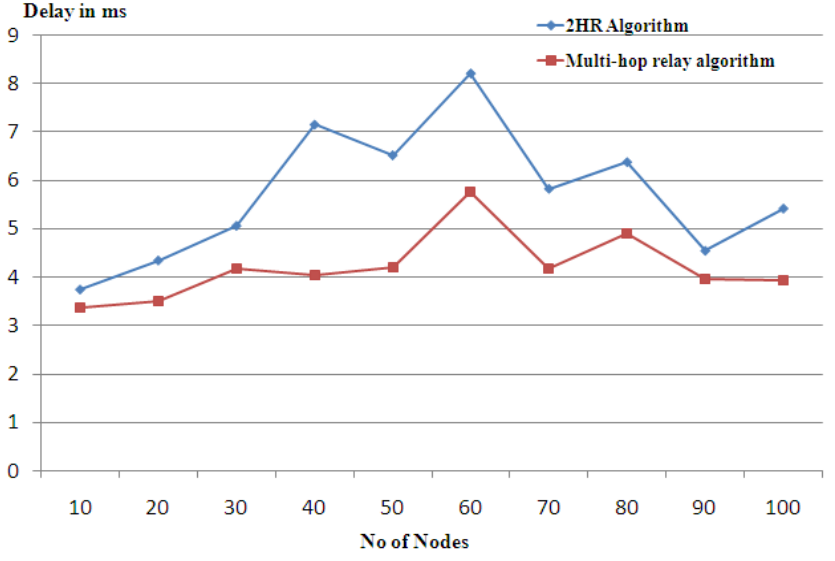

Fig 3 Delay Vs No of Nodes Graph. Compared 2HR routing algorithm with proposed Multi-hop Relay Algorithm

\subsection{Energy Parameter Validation}

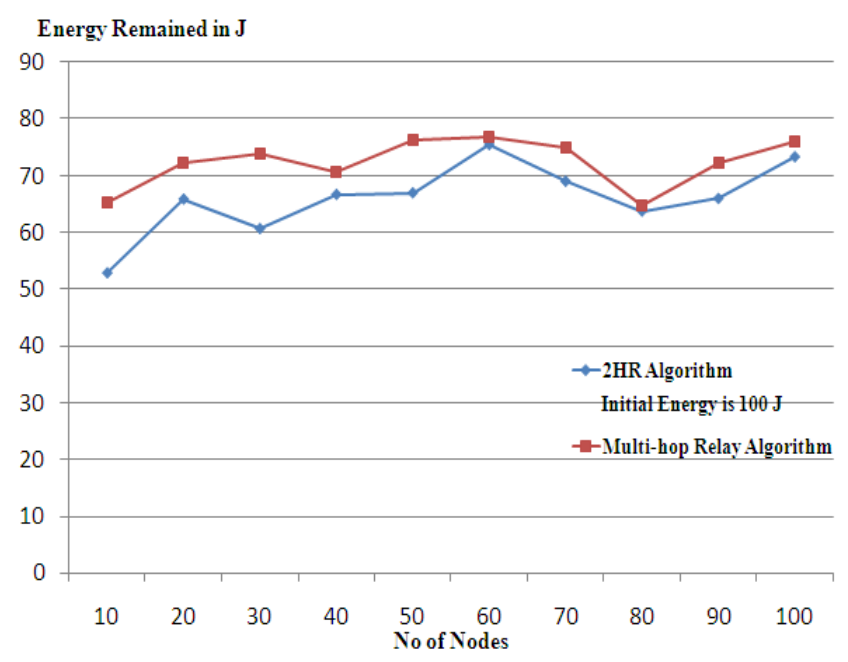

Fig 4 Energy Remained in J Vs No of Nodes Graph. Compared 2 HR routing algorithm with proposed Multi-hop Relay Algorithm

The energy of network remained is calculated by following formula:

Average Energy of network remained $=($ Max energy-Min energy) / 2

A graph is plotted as energy of network against number of nodes. The nodes are varied from 10 to 100 and according to that the average energy of network is calculated for existing $2 \mathrm{HR}$ routing technique and Multi-hop relay technique. The following graph shows that the proposed Multi-hop relay algorithm has optimized average energy up to $77 \%$.

\subsection{PDR Validation}

The energy of network remained is calculated by following formula: $\mathrm{PDR}=$ Number of packets delivered successfully $/$ Total
number of packets sent

The following graph shows that the packet drop obtained by 2HR Routing algorithm is greater than that of proposed Multihop relay algorithm. The PDR optimized by Multi-hop relay algorithm is up to $99.90 \%$

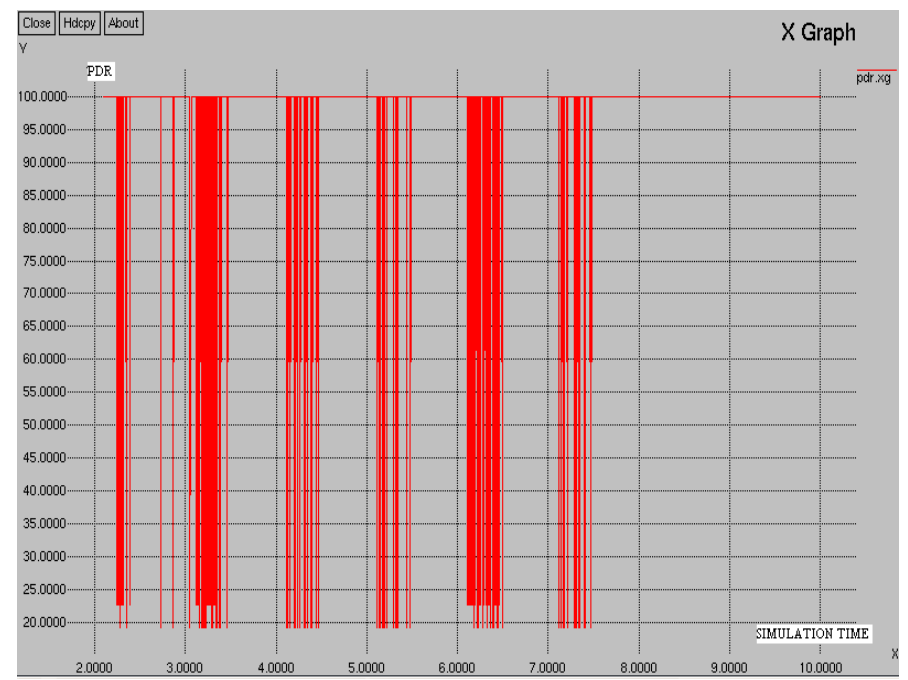

Fig 5 Energy Remained in J Vs No of Nodes Graph. Compared $2 \mathrm{HR}$ routing algorithm with proposed Multi-hop Relay Algorithm

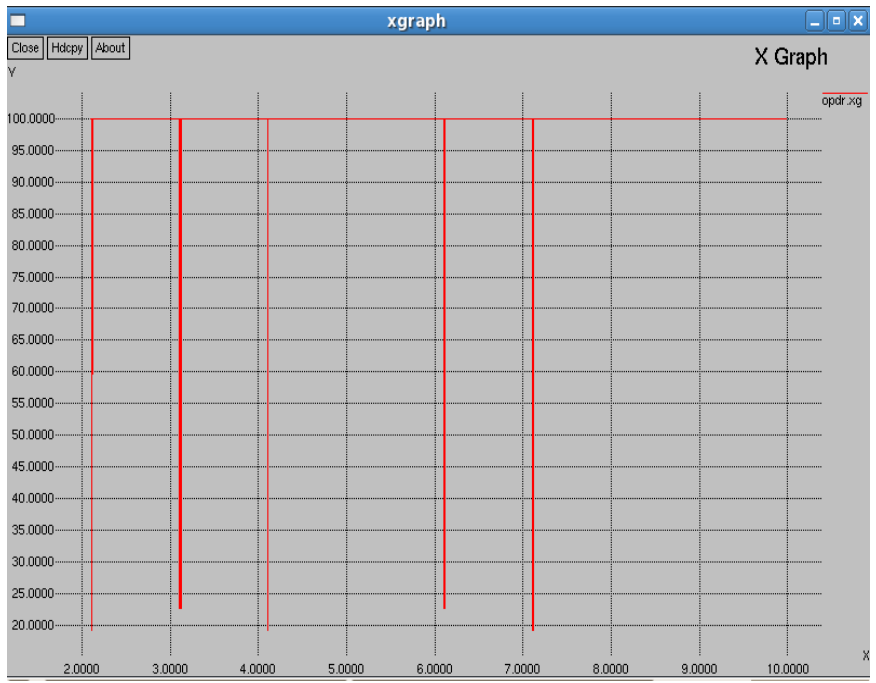

Fig 6 Energy Remained in J Vs No of Nodes Graph. Compared $2 \mathrm{HR}$ routing algorithm with proposed Multi-hop Relay

Algorithm 


\section{CONCLUSIONS}

The proposed multi-hop relay algorithm has been implemented and analyzed by considering delay, PDR and energy consumed in network. The above results shows that this technique has been optimized delay by $3.37 \%$, PDR by $1.36 \%$ and average energy of network by $10 \%$ than previous techniques in dynamic network scenario.

Though this technique has improved the broadcasting efficiency, there is scope to study on link failure mechanism to improve the technique.

\section{ACKNOWLEDGMENTS}

This is my final year ME research project in wireless communication and computing. I would like to express my gratitude to Ms. A. R. Raut who guided me throughout the work. The research work in this paper was supported by G. H. Raisoni College of engineering, Nagpur.

\section{REFERENCES}

[1] D.B. Johnson and D. A. Mallz, "Dynamic source routing in ad hoc wireless networks,"in Mobile Comput, 1996.

[2] C. E. Perkins and E. M. Royer, "Ad-hoc on-demand distance vector routing," in 1999 WMCSA.

[3] Juntao Gaot and Xiaohong Jiangt," Delay Modeling for Broadcast-Based Two-Hop Relay MANETs", 2013 11th International Syrnposiurn and Workshops on Modeling and Optirnization in Mobile, Ad Hoc and Wireless Networks (WiOpt), May 13-17, 2013.

[4] J. Liu, J. Gao, X. Jiang, H. Nishiyama, and N. Kato, "Capacity and delay of probing-based two-hop relay in manets," IEEE Transactions on Wireless Communications, vol. 11, no. 11, pp. 4172-4183, November 2012.

[5] Thomas Kunz, Suranjit Paul, and Li Li, "Broadcasting in Multihop Wireless Networks: the Case for MultiSource Network Coding," IEEE ICC 2012 - Wireless Networks Symposium, 2012.

[6] B.Williams and T. Camp,"Comparison of broadcast techniques for Mobile Ad hoc Networks", Proc.2002ACM MobHoc Conference,June 2002, pp. 194-205.

[7] T. Kunz, S. Paul, and L. Li, "Efficient broadcasting in tactical networks: Forwarding vs. network coding", Proceedings of the 2010 Military Communications Conference (Milcom 2010), San Jose, USA, November 2010.

[8] Sungjoon Park, Wayne E. Stark, "Throughput Analysis of Multi-hop Relaying: the Optimal Rate and the Optimal Number of Hops", 978-1-4673-52390/13/\$31.00 @2013 IEEE.

[9] Wang Gang, Wang Shigang, Liu Cai, Zhang Xiaorong, "Research and realization on improved MANET distance broadcasting algorithm based on percolation theory ," IEEE, 2013.

[10] J. Liu, X. Jiang, H. Nishiyama, and N. Kato, "Generalized two-hop relay for flexible delay control in manets," IEEEIACM Transactions on Networking, vol. 20, no. 6, pp. 1950-1963, December 2012.

[11] P. Li, Y. Fang, J. Li, and X. Huang, "Smooth trade-offs between throughput and delay in mobile ad hoc networks," IEEE Transactions on Mobile Computing, vol. II, no. 3, pp. 427-438, March 2012.

[12] J. Liu, X. Jiang, H. Nishiyama, and N. Kato, "Delay and capacity in ad hoc mobile networks with f-cast relay algorithms," IEEE Transactions on Wireless Communications, vol. 10, no. 8, pp. 2738 - 2751, August 2011.

[13] D. Ciullo, Y. Martina, M. Garetto, and E. Leonardi, "Impact of correlated mobility on delay-throughput performance in mobile ad hoc networks," IEEElACM Transactions on Networking, vol. 19, no. 6, pp. 17451758, December 20 II.

[14] S. Zhou and L. Ying, "On delay constrained multicast capacity of large scale mobile ad-hoc networks," in INFO COM, 2010.

[15] A. Jindal and K. Psounis, "Contention-aware performance analysis of mobility-assisted routing," IEEE Transactions on Mobile Computing, vol. 8, no. 2, pp. 145-161, February 2009.

[16] A. A. Hanbali, R. de Haan, R. J. Boucherie, and J.-K. van Ommeren, "A tandem queueing model for delay analysis in disconnected ad hoc networks," in Proceedings of the 15th international conference on Analytical and Stochastic Modeling Techniques and Applications (ASMTA), 2008, pp. 189-205.

[17] M. J. Neely and R. Urgaonkar, "Optimal backpressure routing for wireless networks with multi-receiver diversity," Ad Hoc Networks, vol. 7, no. 5, pp. 862881, July 2009. 